Research Article

\title{
A Study to Assess the Kowledge, Stress Level and Coping Strategies among Mothers of Child with Type-I Diabetes Mellitus (TIDM)
}

\author{
Ruchika Arora', Bindu Shaiju', Saba Hashmi ${ }^{3}$
}

${ }^{1}$ M.Sc. Nursing Student, ${ }^{2}$ Associate Professor, ${ }^{3}$ Tutor, Rufaida College of Nursing, Jamia Hamdard, New Delhi, India. DOI: https://doi.org/10.24321/2455.9318.201929

\section{I $\quad \mathbf{N} \quad \mathbf{F} \quad \mathbf{O}$}

\section{Corresponding Author:}

Bindu Shaiju, Rufaida College of Nursing, Jamia Hamdard, New Delhi, India.

E-mail Id:

bindushaiju07@yahoo.co.in

Orcid Id:

https://orcid.org/0000-0002-2574-9160

How to cite this article:

Arora R, Shaiju B, Hashmi S. A Study to Assess the Kowledge, Stress Level and Coping Strategies among Mothers of Child with Type-1 Diabetes Mellitus (T1DM). Int J Nurs Midwif Res 2019; 6(4): 28-33.

Date of Submission: 2019-06-14

Date of Acceptance: 2020-03-27

\section{$\begin{array}{llllllll}\mathbf{A} & \mathbf{B} & \mathbf{S} & \mathbf{T} & \mathbf{R} & \mathbf{A} & \mathbf{C} & \mathbf{T}\end{array}$}

Introduction: Type 1 diabetes is a complex and demanding condition, which mainly affects children and places a considerate amount of substantial behavioral and psychological burden on young people and stress to their families.

Objectives: The study aimed to assess knowledge of mothers of T1DM child, to assess the stress level of mothers and to assess their coping strategies adopted while caring for their child with type $1 \mathrm{DM}$.

Methodology: Quantitative (non-experimental) approach with an exploratory survey design was used. The data was collected from 100 mothers of T1DM children at NGO named, Yog Dhyan Foundation, New Delhi. A structured questionnaire and structured rating scale for knowledge and stress assessment respectively and a standardized scale (CRIS) to assess coping strategies of mothers were used for data collection and later data was analyzed using descriptive and inferential statistics.

Result: The findings revealed that $64 \%$ of the mothers had inadequate knowledge and $34 \%$ of the mothers had adequate knowledge regarding Type 1 diabetes mellitus. Majority of the mothers of T1DM children (79\%) had moderate stress, followed by severe stress which was $13 \%$ and only $8 \%$ mothers had mild stress. It was also found that the majority of the mothers of type 1 diabetes mellitus children (84\%) were having above average stress coping ability.

Conclusion: Imparting knowledge to help the parents especially mothers to deal with the problems of the diabetes in children and live a healthy life is pertinent.

Keywords: T1DM, Knowledge, Stress, Coping Strategies, CRIS, Child

\section{Introduction}

World Health Organization defines Diabetes mellitus as a metabolic disorder characterized by chronic hyperglycemia with disturbances of carbohydrate, fat and protein 
metabolism resulting from defects in insulin secretion or its action or both. ${ }^{1}$

Heglar ${ }^{2}$ states" Type 1 Diabetes is a complex and demanding condition, which places a considerate amount of substantial behavioral and psychological burden on young people and stress to their families."

S. Kalra ${ }^{3}$ states, Type 1 Diabetes (T1D) is one of the most common chronic condition among children. Over $50 \%$ of the affected children are living in developing nations, with India being home to an estimated 97,700 children with T1DM that is more than the burden of type 2 diabetes. Every fifth T1DM child is an Indian. The incidence of T1DM is rising $3 \%$ per annum especially in younger children and that too in low prevalent countries. Around 70,000 fresh cases of T1DM are diagnosed every year. This increase in incidence along with enhanced access to insulin and better survival rates will lead to a higher prevalence in the near future.

Kanakatte Mylariah, Prasanna Kumar, ${ }^{4}$ did a study of incidence trends for childhood type 1 diabetes in India estimated that India is housing about 97,700 children with Type 1 Diabetes Mellitus (T1DM). A study of 30 children with insulin-dependent diabetes with age at diagnosis $\leq 15$ years, conducted in 1992, reported a prevalence of $0.26 / 1000$ children. The peak age at diagnosis was 12 years. The first population-based study of prevalence of insulindependent diabetes in South India, shows that insulindependent diabetes is not rare and is higher than that reported from many other Asian countries. The Karnataka state T1DM registry listed an incidence of 3.7/100,000 in boys and 4.0/100,000 in girls over 13 years of data collection. At Karnal, in Haryana, the prevalence of T1DM is $26.6 / 100,000$ in urban and $4.27 / 100,000$ in rural areas of the district, leading to an average prevalence of $10.20 / 100,000$ population. Karnal city has a relatively high prevalence of T1DM (31.9/100,000). An estimated 18,000 children under the age of 15 were newly diagnosed for T1DM in the year 2011 in the above-mentioned regions. The prevalence of T1DM in children is 111,500 according to a World Health Organization report of the International Diabetes Federation for the South-East Asian Region.

Ashok Kumar Das ${ }^{5}$ did a study on Type 1 diabetes in India stating that Type 1 Diabetes Mellitus (T1DM) is also on increase like type 2 diabetes, even though not in the same proportion, but still with a trend of 3-5\% increase/year. India has three new cases of T1DM/100,000 children of 0-14 years. Three sets of prevalence data shows 17.93 cases/100,000 children in Karnataka, 3.2 cases/100,000 children in Chennai and 10.2 cases/100,000 children in Karnal (Haryana).

T1DM may be autoimmune or idiopathic in nature and is present in 9\% cases of insulin deficiency. T1DM is thought of primarily caused by genetic factors, environmental factors, and disorder of the immune regulatory mechanism. A combination of all these three factors causes autoimmune disease, leading to hyperglycemia, ketoacidosis and potentially death, if not treated with insulin. Prediabetes is the phase before the onset of T1DM, which provides a window of opportunity for early intervention. The Indian Council of Medical Research funded Registry of People with Young Diabetes in India (YDR) was started in the year 2006 with 10 collaborating centres across India.

The American Diabetes Association makes specific recommendations for the transfer of responsibility for treatment management, noting that school-age children (ages 8-11 years) can begin to assume more tasks with regard to taking care of their diabetes, which has actually become a part of their body, such as insulin injections/ boluses and blood glucose monitoring, but they continue to need significant assistance and supervision when making management decisions. Adolescents (ages 12 years and older) are able to perform most of the tasks of diabetes management on their own, but they still need help with decision making regarding insulin adjustments. Hence the diabetic children always require attention and supervisions of the caretaker to have better health outcomes.

Stress can accumulate over time and may require intervention. One type of stress that can become longterm is that which people feel as a result of parenting a child. This type of stress is critical to understand due to the link between negative parent and child outcomes that can result into the symptoms like loss of interest in working, feeling low, frustrated at times, not able to do any task well, not able to give time to other activities other than child's disease management. Being a parent can be one of life's most joyful and the best experiences, but at times, in everyone's life there are demands and aggravations of daily living which leads to stress. Due to additional stress of caring for children, at times, make parents feel angry, anxious, or simply "stressed out". These tensions are a normal, inevitable part of family life, and parents need to learn ways to cope so that they don't feel inundated by them.

Moreover, beyond the challenges of daily care and monitoring, living with the constant threat of health deterioration and future complications, the young patients face emotional and psychological difficulties that reflect on their own coping as well as their social circle and family.

Addressing parental stress and coping early in the diagnostic process and providing continued support may decrease the effects of parental stress that can have on the child who is diabetic and on their insulin therapy and the family. 
J. Silverstain, ${ }^{6}$ delineated in the study about caring for children with diabetes, professionals need to understand the importance of involving adults in the child's diabetes management. Young children including the school aged children are unable to take their own diabetic care and middle school and high school students should not be expected to take care of their own diabetes management independently. Thus the parents need to put forward their proactive steps in child care management and be educated about how to care for a child and adolescent with diabetes. The entire family unit should be involved in care.

\section{Materials and Methods}

The research approach selected for the study was quantitative, non experimental. An exploratory survey design was used to synthesize research findings concerning the knowledge, stress level and coping strategies used by the mothers of type 1 diabetes mellitus children. A total of 100 mothers of T1DM children, attending the NGO named, Yog Dhyan Foundation, in New Delhi, were selected by purposive sampling technique. Samples who met the inclusion criteria i.e mothers of children having T1DM falling under the age group of 7-18 years, mothers of children having T1DM since 1 year and mothers who were available during the data collection period and attending Yog Dhyan Foundation were enrolled for the study. There was no attrition and contamination of sample. The tools used for data collection comprised of four sections. Section-A demographic characteristics of the mothers of T1DM children consisting of 15 items. Section-B consisting of 25 questions related to knowledge of the mothers on TIDM on 3 areas i.e general questions related to T1DM, impact of illness on health and management aspect of T1DM. Section- $C$ consisting of structured rating scale to assess the stress level of mothers of T1DM child which consisted of 20 questions. Section-D consisting of standardized Coping Resource Inventory for Stress (CRIS) ${ }^{7}$ which consisted of 32 questions to assess the coping strategies used by the mothers of T1DM child. The tools were validated by giving it to 7 experts from the field of Pediatrics, psychiatry and Nursing. The reliability of the tools were established using KR-20 for knowledge questionnaire and Cronbach alpha for the stress inventory and with $r=0.85$ the tool was proved to be reliable. Scoring was done by dividing the scores into different categories as reflected in Table-1, table- 2 and table-3. After obtaining ethical permission from the IEC and permission from the administrative authority of Yog Dhyan foundation, the selected sample was informed about the procedure of data collection and their informed consent was taken. The tools were administered on the sample and the data collected were analyzed using descriptive and inferential statistics.
Table I.Categories of the knowledge score and the range of score

\begin{tabular}{|c|c|}
\hline Categories & Range of Score \\
\hline $\begin{array}{c}\text { Inadequate Knowledge } \\
(\leq 75 \%)\end{array}$ & $0-19$ \\
\hline Adequate Knowledge $(>75 \%)$ & $20-25$ \\
\hline
\end{tabular}

Table-2.Scoring of the Stress Scores

\begin{tabular}{|c|c|}
\hline Score & Level of Stress \\
\hline 0 & No Stress \\
\hline $1-26$ & Mild Stress \\
\hline $27-53$ & Moderate Stress \\
\hline $54-80$ & Severe Stress \\
\hline
\end{tabular}

Table 3.Scoring of the Coping Scale

\begin{tabular}{|c|c|}
\hline Overall Coping Score & Interpretation \\
\hline An overall score of $(>3.5)$ & $\begin{array}{c}\text { Superior Stress-Coping } \\
\text { ability }\end{array}$ \\
\hline An overall score of $(2.5-3.4)$ & $\begin{array}{c}\text { Above Average Stress- } \\
\text { Coping ability }\end{array}$ \\
\hline An overall score of (1.5-2.4) & $\begin{array}{c}\text { Average Stress-Coping } \\
\text { ability }\end{array}$ \\
\hline An overall score $(<1.5)$ & $\begin{array}{c}\text { Below Average Stress } \\
\text { Coping ability }\end{array}$ \\
\hline
\end{tabular}

\section{Result}

\section{Section I: Description of Sample Characteristics}

The study findings revealed that out of the 100 mothers of T1DM children, majority (46\%) of the mothers were of age group (31-38 years), with regard to occupation of mothers it was found that majority (76\%) were housewife; majority of the mothers (84\%) were Hindu and (14\%) of the mothers were (Muslims), with regard to the educational qualification of mothers of T1DM children, (34\%) had primary education $\&(27 \%)$ had Secondary Education while (20\%) were postgraduate and (14\%) were graduate. Almost similar percentage $36 \% \& 31 \%$ of the mothers have monthly family income of (Rs. 7000 to 10000) \& (Rs. 10001 - 15000) respectively. (55\%) lived in nuclear family and $(40 \%)$ of the mothers lived in joint family; most of them (81\%) were not having any family history of diabetes mellitus and only (19\%) have history of diabetes mellitus; mothers who had 3 children were (35\%), from which (95\%) mothers had a single child with T1DM. When we draw our attention to the age group of T1DM children, (35\%) were in the age 
group of (10-12 years), followed by (24\%) were in the age group of (13-15 years). In relation to the age of onset of T1DM children, almost equal percentage of children that is $(46 \%)$ and $(44 \%)$ has the age of onset as (6-10 years) and (1-5 years) respectively. With regard to gender of a child, (52\%) children with T1DM were females and remaining $(48 \%)$ were males.

Table 4.Frequency and percentage distribution of mothers of children with TIDM by their level of knowledge

\begin{tabular}{|c|c|}
\hline Level of Knowledge & $\begin{array}{c}\mathrm{n}=100 \\
\text { Frequency/ } \\
\text { Percentage }\end{array}$ \\
\hline Adequate Knowledge (20-25) & 36 \\
\hline Inadequate knowledge (0-19) & 64 \\
\hline
\end{tabular}

\section{Section2: Findings related to knowledge of the mothers on type I diabetes mellitus (TIDM)}

The data depicted in table 4 , shows that $64 \%$ of the mothers had inadequate knowledge, and over $36 \%$ of the mothers had adequate knowledge on type 1 diabetes mellitus and management of child with T1DM.

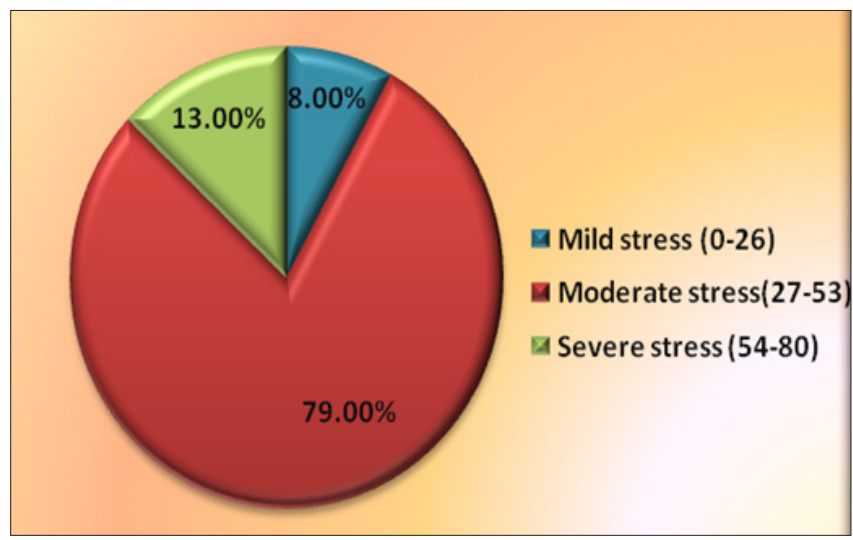

Figure I.Pie diagram showing the Frequency/ Percentage Distribution of mothers with TIDM child by their Level of Stress

Section 3.Findings related to the assessment of the stress levels of the mothers in caring for child with Type I Diabetes Mellitus (TIDM)

The data depicted in figure 1 , shows that majority of the mothers of type 1 diabetes mellitus children had Moderate Stress of about $(79 \%)$ and followed by the Severe Stress which was (13\%) and only (8\%) had Mild Stress.

Section 4.The findings related to coping strategies used by mothers of type 1 diabetes mellitus child (T1DM).

The data depicted in figure 2 , shows that majority of the mothers of type 1 diabetes mellitus children are having

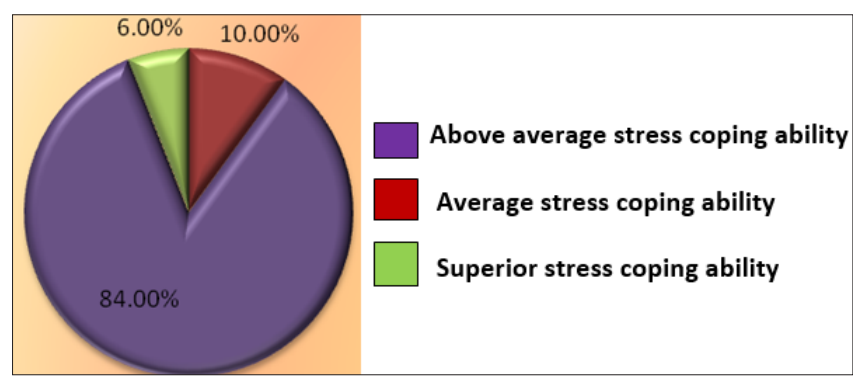

Figure 2.Pie diagram showing the Frequency/ Percentage distribution of mothers by their coping strategies

Above Average stress coping ability with (84\%) followed by Average Stress coping ability with (10\%) and only (6\%) mothers fall in the Superior Stress coping ability. None were in the below average stress coping ability.

\section{Discussion}

The study findings showed that $64 \%$ of the mothers had inadequate knowledge and over $36 \%$ of the mothers had adequate knowledge on type 1 diabetes mellitus and management of child with T1DM.

The findings of the study are closely similar to the study conducted by Othman ${ }^{8}$ which showed that although mothers of pediatric type-1 diabetic children have the knowledge of DKA, $54 \%$ had adequate knowledge and $46 \%$ patients had inadequate knowledge. Out of which (33\%) responded that they do not know what is DKA (Diabetes Ketoacidosis). However they need further education from the diabetes health educators and diabetes care team as most of them do not know about the sick day management.

Another study contrary to present study was, by Amorim de Pedrosa $\mathrm{K},{ }^{9}$ which revealed that with regard to knowledge of caregivers, $72 \%$ had a satisfactory score. Most of the caregivers could perform the treatment (80\%), and $(24 \%)$ did not trust their skill; $(72 \%)$ of respondents could recognize hyperglycemia and (96\%) could recognize hypoglycemia, (80\%) could apply the correct technique of insulin administration; (80\%) managed to keep the recommended diet.

The present study findings showed that majority of the mothers of type 1 diabetes mellitus children had Moderate Stress of about (79\%), followed by the Severe Stress which was (13\%) and only (8\%) had Mild Stress.

Kaitlyn Rechenberg, ${ }^{10}$ did a study on stress and PTSS (Post Traumatic Stress Symptoms) and found both were prevalent in mothers of youth with T1DM. While PTSS was most severe at disease onset, symptoms often persisted 1 to 5 years after diagnosis. The diagnosis of T1D in a child was traumatic for mothers.

Study done by Robin Whitmore, ${ }^{11}$ found that the prevalence of parental psychological distress with an average of 
$33.5 \%$ of parents reporting distress at diagnosis and $19 \%$ of parents reporting distress 1 to 4 years after diagnosis. This Longitudinal study demonstrated a slight decrease in distress over time. The prevalence of anxiety symptoms ranged from $21 \%$ to $59 \%$, depressive symptoms from $10 \%$ to $74 \%$, psychological distress from $29 \%$ to $33 \%$ and symptoms of PTSD from $19 \%$ to $24 \%$ in parents of children with T1DM.

Study done by Streisand, ${ }^{12}$ examined data on parental demographics, stress, coping, psychological well-being, and physical well-being from a national dataset. A subset of 278 mothers who had children diagnosed with diabetes (either type 1 or type 2; M age=12.1; 46\% female children; $78 \%$ white) were included in the analyses for the current study. Results suggested that mothers of non-white children and mothers from single parent households experience poorer psychological and physical well-being than mothers of white children or mothers with a partner.

M. Grey, ${ }^{13}$ had suggested that parents worry about the lack of cooperation on the part of their children when they reach adolescence and thereafter. Several studies have indicated that psychological factors (such as stressors and coping patterns) that arise during adolescence are often associated with negligence in their self-management. Moreover, findings have revealed that a diabetic child's gender affects child-parent cooperation and parental coping.

The researcher could not substantiate and compare the findings with studies done in India as studies on psychological aspects of caregivers of type I DM child were not available in the Indian context.

\section{Conclusion}

On the basis of results of data analysis, the following salient conclusions were drawn. Many of the mothers were unaware about the type 1 diabetes mellitus in children; moreover, majority of mothers were moderately stressed in caring for the child's diabetes, its impact of illness on health aspects and management of child's diabetes and most of the mothers had favorable coping towards their child's diabetes and were above average stress coper. Addressing parental stress and coping early in the diagnostic process and providing continued support may decrease the effects of parental stress that can have on the child who is diabetic. The community health nurse can interact with the family, initiate the family health promotion and assist families and their members to get the knowledge about type I DM and its complications and management in children as well as adults.

\section{Acknowledgement}

I express my deep sense of gratitude and indebtedness to my mentor Ms. Bindu Shaiju Associate Professor, Rufaida college of Nursing, for their support, esteemed guidance, during my research endeavor. Words cannot express my very profound gratitude and love to my parents Mr. Om Prakash Arora, Mrs. Anju Arora, and my brother Mr. Vivek Arora for their unconditional love and support. I am profoundly indebited to all experts who contributed their time and efforts towards validating and refining tools used in present study.

\section{Conflicts of Interest: None}

\section{References}

1. WHO, Definition, diagnosis and classification of diabetes and its complications. 1st ed. USA; 1999.

2. Hagger V, Trawley S, Hendrieckx C, Browne JL, Cameron $F$, Pouwer $F$ et al . Diabetes MILES Youth-Australia: methods and sample characteristics of a national survey of the psychological aspects of living with type 1 diabetes in Australian youth and their parents. BMC Psychology 2016; 4(1): 42. Available from: https:// bmcpsychology.biomedcentral.com/articles/10.1186/ s40359-016-0149-9 [PubMed/ Google Scholar].

3. Kalra S, Azad K, Prasanna Kumar K, Zabeen B. Type 1 diabetes in children: Fighting for a place under the sun. Indian Journal of Endocrinology and Metabolism 2012; 16(1): S1-S3. [PubMed/ Google Scholar].

4. Prasanna Kumar K. Incidence trends for childhood type 1 diabetes in India. Indian Journal of Endocrinology and Metabolism 2015; 19(7): 34. [PubMed/Google Scholar].

5. Das AK. Type 1 diabetes in India: Overall insights. Indian Journal of Endocrinology Metabolism 2015; 19(1): S31-S33. [DOI: 10.4103/2230-8210.155372/ PubMed/ Google Scholar].

6. Silverstein J. Care of children and adolescents with type 1 diabetes. St Alexandria, VA; 2005. Available from: https://care.diabetesjournals.org/content/28/1/186.

7. Matheny K, Aycock D, Curlette W, Junker G. The Coping Resources Inventory for Stress: A measure of perceived resourcefulness. Journal of Clinical Psychology 2003; 59(12): 1261-1277. [Google Scholar/ ResearchGate].

8. Othman A, Awwad MA, Aziz KMA, Asiri SM, Alqahtani NM. Knowledge and Awareness of Mothers about Diabetic Ketoacidosis among Type-1 Diabetic Children and Their Action and Response in Emergency Conditions in Aseer Region of Saudi Arabia. Journal of Diabetic Complications \& Medicine 2018; 3(2): 122. Available from: https:// www.semanticscholar.org/paper/Knowledge-andAwareness-of-Mothers-about-Diabetic-OthmanAwwad/ed9860ee6ec001076f4f2c23f3efc3fd42250511 [Google Scholar].

9. Amorim de Pedrosa K. Education effectiveness in diabetes mellitus type 1 management made by children of caregivers. Brazil; 2016.

10. Rechenberg K, Grey M, Sadler L. Stress and Posttraumatic Stress in Mothers of Children With Type 
1 Diabetes. Journal of Family Nursing 2017; 23(2): 201225. [DOI: 10.1177/1074840716687543/ PubMed/ Google Scholar].

11. Whittemore R, Jaser S, Chao A, Jang M, Grey $M$. Psychological Experience of Parents of Children With Type 1 Diabetes. The Diabetes Educator 2012; 38(4): 562-579. [PubMed/ Google Scholar].

12. Streisand R, Mackey ER, Herge W. Associations of parent coping, stress, and well-being in mothers of children with diabetes: Examination of data from a national sample. Maternal and Child Health Journal 2010; 14: 612-617. Available from: https://psycnet.apa.org/ record/2010-14060-014 [Google Scholar].

13. Grey $M$, Jaser $S$, Whittemore R, Jeon S, Lindemann E. Coping Skills Training for Parents of Children With Type 1 Diabetes; 12-Month Outcomes. Nursing Research. 2011; 60(3): 173-181. [PubMed/ Google Scholar]. 